\title{
New protocol for pathological diagnosis and treatment pathway based on the "BIOCO" collector tool in Henan Cancer Hospital
}

Wenqun Xing ${ }^{1}$, Wenjia Tian ${ }^{1}$, Bing wei ${ }^{1}$, Jiani Yuan ${ }^{1}$, Lingyue Xing ${ }^{1}$, Chengzhi Zhao ${ }^{1}$, Yuxi Chang ${ }^{1}$, Haibo Sun ${ }^{1}$, Rui Sun ${ }^{1}$, Zhongxian Zhang ${ }^{1}$, Yang $\mathrm{Yu}^{1}$, and Jie Ma ${ }^{1}$

${ }^{1}$ Affiliation not available

July 13, 2020

\begin{abstract}
Background. 2018 Guideline from the College of American Pathologists(CAP), the International Association for the Study of Lung Cancer (IASLC), and the Association for Molecular Pathology(AMP) established a benchmark turnaround time (TAT), according to which the results were supposed to be available to the treating oncologist within 10 working days. Methods. In this study, we established and validated a new protocol for pathological diagnosis and treatment pathway that was tested in the Henan Cancer Hospital, China. Results. Compared with the routine pathway, the new path adopts a parallel approach to conventional pathology and molecular pathology, which significantly shortens the TAT to 4-6 days. Self-designed DNA biology collector "BIOCO" tool, designed by our team to collect specimens that are discarded when the wax block is trimmed, can effectively save pathological samples, avoid cross-contamination and reduce the period extension caused by re-sampling. Most importantly, its accuracy and effectiveness are consistent with conventional collection methods. Conclusions. Therefore, the new diagnosis and treatment pathway based on the BIOCO collector can be used as a practical approach to the molecular diagnostic platform of the hospital pathology department.
\end{abstract}

New protocol for pathological diagnosis and treatment pathway based on the "BIOCO" collector tool in Henan Cancer Hospital

Wenqun Xing ${ }^{*}$, Wenjia Tian ${ }^{3 *}$, Bing $\mathrm{Wei}^{2}$, Jiani Yuan ${ }^{3}$, Lingyue Xing ${ }^{3}$, Chengzhi Zhao $^{2}$, Yuxi Chang ${ }^{2}$, Haibo Sun ${ }^{1}$, Rui Sun ${ }^{2}$, Zhongxian Zhang ${ }^{2}$, Yang $\mathrm{Yu}^{3 \#}$, Jie Ma ${ }^{2 \#}$

* These authors contributed equally to this work.

\# corresponding author

\section{Affiliations:}

${ }^{1}$ Department of Thoracic Surgery, Henan Cancer Hospital, Zhengzhou, Henan, China.

${ }^{2}$ Department of Pathology, Henan Cancer Hospital, Zhengzhou, Henan, China.

${ }^{3}$ Novogene Co., Ltd., Beijing, China.

\# Correspondence:

Yang $\mathrm{Yu}$

Novogene Co., Ltd., Beijing, China. 
Jiuxianqiao North Road, Chaoyang District, Beijing 100016, China.

E-mail: yuyang@novogene.com

Jie Ma

Department of Pathology, Henan Cancer Hospital, No.127, Dongming Road, Zhengzhou, Henan 450003, China.

E-mail: majie_hnzl@163.com

\title{
Synopsis:
}

In order to save the time of tumor NGS detection cycle, Pathology Department of Henan Cancer Hospital developed an optimized diagnosis and treatment pathway. Combined with the small sample collector "BIOCO", the new pathway can reduce the patient's detection waiting time by $4-6$ days and won valuable treatment time for patients.

\section{Data Availability Statement}

The data used to support the findings of this study are available from the corresponding author upon request.

\begin{abstract}
Background . 2018 Guideline from the College of American Pathologists(CAP), the International Association for the Study of Lung Cancer (IASLC), and the Association for Molecular Pathology(AMP) established a benchmark turnaround time (TAT), according to which the results were supposed to be available to the treating oncologist within 10 working days. Methods. In this study, we established and validated a new protocol for pathological diagnosis and treatment pathway that was tested in the Henan Cancer Hospital, China.Results . Compared with the routine pathway, the new path adopts a parallel approach to conventional pathology and molecular pathology, which significantly shortens the TAT to 4-6 days. Self-designed DNA biology collector "BIOCO" tool, designed by our team to collect specimens that are discarded when the wax block is trimmed, can effectively save pathological samples, avoid cross-contamination and reduce the period extension caused by re-sampling. Most importantly, its accuracy and effectiveness are consistent with conventional collection methods. Conclusions. Therefore, the new diagnosis and treatment pathway based on the BIOCO collector can be used as a practical approach to the molecular diagnostic platform of the hospital pathology department.
\end{abstract}

Keywords: New diagnosis and treatment pathway; TAT; "BIOCO" collector

\section{Introduction}

With the widespread application of Next Generation Sequencing (NGS) molecular pathology in clinical work, a large number of genetic aberrations have been identified, thus permitting the development of new targeted therapy for patients with cancer, including an advanced non-small cell lung cancer (NSCLC) ${ }^{1}$. Over recent years, new-targeted drugs, molecular markers, and detection methods have been constantly emerging ${ }^{2}$. For example, the recent treatment guidelines of the National Comprehensive Cancer Network (NCCN) in the United States has suggested that nine driving genes (EGFR/KRAS/HER2/ALK/ROS1/MET/BRAF/RET/NTRK) should be routinely detected in lung cancer to provide more detailed genetic mutation information for the precise treatment of lung cancer ${ }^{3}$.

As an advanced genetic testing method, NGS has the advantages of high sensitivity and comprehensive detection. However, compared to traditional methods like fluorescence in situ hybridization (FISH), immunohistochemistry (IHC), and reverse transcription-polymerase chain reaction (RT-PCR), NGS has longer detection cycle (results are usually obtained within 4-10 natural days). Recent guideline recommendations by AMP, CAP, and ASLC established a benchmark turnaround time (TAT). Based on the guideline, the 
results were supposed to be available to the treating oncologist within 10 working days. ${ }^{4,5}$. In a retrospective analysis of all NSCLC cases within one year from a large academic institution, DiStasioet al found that $81.5 \%$ of the test results were presented within ten working days as recommended by the guidelines, which proved the feasibility of TAT recommended by CAP, IASLC, and AMP guideline ${ }^{6}$.

With the development of precision medicine, both patients and clinicians expect to benefit from precise timely treatment, so a rapid and accurate diagnosis and genotyping workflow for lung cancer are required. Currently, many hospitals are still using routine clinical diagnosis and treatment pathways, which imply TAT of about 10-16 days. In this study, we established and validated a new protocol for pathological diagnosis and treatment pathway that was tested in the Henan Cancer Hospital, China.

The current protocol in Henan Cancer Hospital relays on two primary specimen sources: 1) in-hospital analyses and delivery of the specimen; 2) analyses and delivery of the specimen from Henan Cancer Hospital branches. The puncture specimens from the hospital undergo paraffin embedding, sectioning, hematoxylineosin (H\&E) staining, IHC \pm FISH, and NGS detection in sequence, which take approx.7-16 days for the entire molecular pathology detection of lung cancer (Figure 1a ). H\&E staining, morphological diagnosis and release of the test report require approximately 1-3 days. The molecular pathology testing for lung cancer can be started only when the H\&E report is sent back to the Department of Molecular Pathology. Consequently, 5-8 natural days are required for NGS testing, which is then sent back by mail to the clinic. Above all, it takes 8-14 natural days for the FFPE specimens from the consortium, the shipment by routine diagnosis and treatment pathway to issuing of the molecular pathological report of lung cancer (Figure 1b ). Through routine detection pathways, a detection cycle for some patients in the hospital lasts about 15 days, while some get discharged, which prevents them from timely obtaining the treatment plan or even makes them miss the best treatment time. Therefore, the optimization of the diagnosis and treatment pathway, and shortening of the detection cycle have become issues of the utmost importance.

During the actual operation of the lung-cancer based on 6 genes NGS testing kit (Novogene Co., Ltd., Beijing, China) in hospital, we found that the main factors affecting TAT included: specimen type, transport link, clinical diagnosis and treatment pathway, molecular pathology detection duration and report release route. In response to the above problems, Henan Cancer Hospital proposed a new diagnosis and treatment protocol. For the cycle problem, we used the Life sequencing platform, which has the fastest detection cycle compared to other sequencers. At the same time, we optimized the diagnosis and treatment path to shorten unnecessary waiting times in the process. For the problem of insufficient sample size, we designed a small sample collector "BIOCO", which is used to collect waste samples when constructing wax blocks, and as a supplement when the sample is insufficient. The new clinical diagnosis and treatment approach based on Life sequencer and BIOCO collector for public hospitals can provide patients with more timely test reports and accurate treatment.

\section{Materials and Methods}

\section{New Clinical Diagnosis and Treatment Pathway}

TAT refers to the time from when samples are received by pathology laboratory to when the test results are sent back to the clinicians ${ }^{4,5}$. In order to ensure better treatment decisions for patients in a timely manner, histopathological diagnosis, clinical staging, and molecular typing of lung cancer must be carried out quickly and efficiently. The Molecular Pathology Department of Henan Cancer Hospital summed up experience during the operation using the NGS platform and tested the pattern of parallel development in routine pathological diagnosis and molecular pathological diagnosis, developing new and more efficient diagnosis and treatment pathway with shorter TAT that are suitable for public hospitals. The protocol (Figure 2 ) is presented as follows:

Day 1: according to the relevant examinations and preliminary diagnosis of patients (medical history, routine blood testing, blood coagulation test, electrocardiogram, tumor markers testing, and a contrast chest computed tomography data), clinicians propose a pathological and genetic diagnosis ${ }^{7}$. The patient's puncture 
specimen is sent to the pathology laboratory for registration and entry system. The molecular pathology department checks the doctor's recommendation, communicates with the patient, signs the supplementary application form for lung cancer-related 6 genes testing, and confirms the pathological diagnosis and genetic diagnosis prescribed at the same time.

Day 2: puncture specimens in the pathology department are fixed, dehydrated, embedded in paraffin, cut into the paraffin section, and stained with H\&E. At the same time, part of the sample is sent to the molecular pathology department by the medical coordinator. At the department of pathology, the diagnosis of IHC and FISH is judged according to the preliminary pathological diagnosis results. If further diagnosis of IHC or FISH is needed, the pathological results are uploaded into the electronic system on the 6th day, and the clinicians are informed. BIOCO paraffin rolls and unstained white paraffin slides are sent to the Department of Molecular Pathology, checked and registered in the system. BIOCO samples and white films, which are sent to the Department of Molecular Pathology, are also subjected to H\&E staining and morphological diagnosis, and the results are checked. When the tumor cell content is greater than $10 \%$, the next detection step is performed.

Day 3-7: NGS can deliver results in the fastest 4 days. on the third day, tissue specimens are dewaxed and digested, and DNA and RNA extraction are performed, followed by RNA reverse transcription. Library construction is done on day 4; after RNA reverse transcription, the cDNA and extracted DNA are amplified by multiple PCR, followed by primer digestion, amplicons ligation, magnetic beads purification, and library quantification. Consequently, emulsion PCR will be performed. On day 5, templates enrichment, sequencer cleaning, on-line sequencing, and automatic analysis of biological information are conducted. The generation and check of lung cancer-related 6 genes tests are performed on day 6 . The report is then fed back to the clinic on day 7. If there is insufficient sample size or other circumstances, the NGS detection period will be extended.

For small biopsy specimens in our hospital, the clinicians prescribe the lung cancer related 6 genes test that can be done within 1 day and NGS test that takes 4-7 days. The test report is reviewed and fed back to the clinic in one day. So the entire process lasts 6-9 days. Clinicians make treatment plans according to the test results for the patient harboring specific mutations, referring to NCCN Guidelines and Chinese Guidelines.

\section{Real-world TAT data obtained by using new diagnostic and therapeutic pathway}

The NGS platform used in the hospital is DA8600. The platform is based on the principle of semiconductor sequencing and is the first domestic sequencer approved by National Medical Products Administration (NMPA) for tumor genetic testing. Its main advantages are small sample requirements, short testing period, and moderate throughput. Therefore, the platform is integral part of the novel diagnosis and treatment pathway.

"Human EGFR, KRAS, BRAF, PIK3CA, ALK and ROS1 Gene Mutation Detection Kit (semiconductor sequencing)" (Novogene Co., Ltd., Beijing, China) were used to detect lung cancer-related 6 genes. From January 1, 2019 to November 30, 2019, 384 specimens of lung cancer-related 6 genes tests were detected on the hospital platform, all of which were puncture biopsy specimens. The lung cancer-related 6 genes test was carried out by the new clinical diagnosis and treatment pathway in the hospital. The proportion of in-hospital reports with TAT $<5$ days was $72.40 \%$, and the report with TAT of 5-7 days was $14.84 \%$. The test report with TAT within 7-10 days was $12.76 \%$. Therefore, all reports could be issued within 10 days, which gives additional 4-6 days for patients treatment compared with a routine pathway(Table 1).

\section{Small puncture specimens collector "BIOCO"}

In our hospital, most of the specimens for genetic testing in clinic are small biopsy specimens. In addition, the samples used for genetic testing are usually the remaining material from the routine pathological diagnosis, so, sometimes, sample size or the amount of extracted DNA may be insufficient. Through the analysis of the above real-world data, we found that the reasons for TAT $>5$ days mainly include insufficient specimens 
in the initial stage of the project or samples being re-send due to failed extraction of nucleic acid. To solve these problems, the new type biopsy specimen collector $\mathrm{BIOCO}^{\mathrm{TM}}$ was designed.

Design principle and device diagram of BIOCO collector

BIOCO was made of polyvinyl chloride (PVC) material using a one-time pressing (patent application number is 201820902335.6). It is a small device used to collect waste sample during sectioning; the collected samples can be used as a supplement when there are insufficient samples.Figure 3 shows a schematic diagram of BIOCO from different angles. The BIOCO tool consists of two round magnets on the collector that can be adsorbed on the microtome blade holder, thus making it to move arbitrarily. Before slicing, the prepared FFPE specimen is fixed on the microtome. The BIOCO collector is then adsorbed on the knife holder, and the leading edge is aligned with the knife. When slicing, the cut tissue will directly enter the collector, without contaminating the knife holder. After the specimen enters the collector, the upper cover with a buckle is fastened to the collector, and the cover is numbered with a marker. The specimen can be processed in the laboratory, after which samples will be stored or shipped. When the laboratory specimen processing starts, the lower piston of the collector is pull out and tightly connected to the $1.5 \mathrm{~mL}$ centrifuge tube, the upper cover is flicked to make the wax roll enter the centrifuge tube. If there is a residual specimen on the wall, the tube connecting the collector is vertically placed on the test tube rack, dewaxing liquid is directly added into the collector, and specimen is dissolved into the centrifuge tube for nucleic acid extraction.

\section{Comparison between BIOCO and Routine Collection (ROUCO) method}

Randomly selected biopsy specimens were collected from 163 patients with advanced NSCLC between January 1, 2019 and November 30, 2019. At the same time of H\&E sectioning, each specimen was collected by the BIOCO method and stored until further analysis. When the patient was diagnosed with NSCLC, the ROUCO collection method was used to obtain paraffin samples from the same patient. Finally, 39 paired specimens were obtained for subsequent experiments. DNA extraction was performed using QIAamp DNA FFPE Tissue Kit (Qiagen, Germany, \#56404) and DNA concentration; the quality was determined using Nanodrop2000 and Qubit. Quantitative mutation detection was carried out using ACCBio's human EGFR gene mutation quantitative detection kit (Beijing ACCB Biotech Ltd. \#180626). SPSS software (version 19.0, SPSS Ins) was used to analyze the data from the two groups of samples. A P value $<0.05$ was considered to be statistically significant.

\section{Results}

\section{DNA extraction efficiency between BIOCO and ROUCO}

When using the Nanodrop2000, the median DNA concentration of the samples collected by BIOCO was $13.8 \mathrm{ng} / \mu \mathrm{l}(1.4-68.5 \mathrm{ng} / \mu \mathrm{l})$; the median DNA concentration measured by the ROUCO method was $25.7 \mathrm{ng} / \mu \mathrm{l}$ $(2.5-200 \mathrm{ng} / \mu \mathrm{l})$. When using the Qubit test, the median DNA concentration of the samples collected by BIOCO was $3.5 \mathrm{ng} / \mu \mathrm{l}(0.3-34.0 \mathrm{ng} / \mu \mathrm{l})$, while the median DNA concentration measured by the ROUCO method was $4.3 \mathrm{ng} / \mu \mathrm{l}(0.2-27.0 \mathrm{ng} / \mu \mathrm{l})$. There was no significant difference $(\mathrm{p}>0.05)$ in DNA extraction efficiency between BIOCO group and ROUCO group under the same elution volume (Figure 4 ), which suggested that BIOCO could meet the needs of subsequent testing.

\section{The equivalent experiment of pollution prevention by BIOCO and ROUCO method}

Ten paraffin blocks with a known high abundance of EGFR exon 21 L858R mutation (A1-10) and 10 paraffin blocks (B1-10) of EGFR wild type specimens were selected. Alternate section of mutant and wild-type paraffin blocks (A1-B1-A2-B2-A3-B3..........A10-B10) was performed. ROUCO method was used for the A1-10, and BIOCO method for B1-10. Each paraffin block was replaced with disposable blades and tweezers before slicing, and the tool holder was not processed. The cut paraffin was collected into a 1.5 $\mathrm{mL}$ centrifuge tube, and DNA was extracted from all 20 specimens according to the standard nucleic acid extraction process, followed by qualitative detection of EGFR gene by fluorescence quantitative PCR. As shown in Table $\mathbf{2}$, all samples obtained the expected results, and no cross-contamination occurred. 


\section{Extraction of DNA by BIOCO and ROUCO for EGFR mutation detection}

A total of 39-paired specimens were tested for EGFR by amplification refractory mutation system (ARMSPCR), and the positive rate was $51.3 \%$ in both groups (Table 3 ). The results were completely consistent in the mutant subtypes of EGFR between the two groups. We defined 0-10\% as low abundance, 10-20\% as medium abundance, $>20 \%$ as high abundance. The consistencies of mutation with high abundance, medium abundance, and low abundance in the two groups were $85.7 \%, 100 \%$, and $83.3 \%$, respectively. Although the two patients' abundance results were inconsistent, the difference between the two groups was not obvious $(\mathrm{P}>0.05)$.

\section{Discussion}

The new diagnosis and treatment pathway at our hospital is based on the Thermo DA8600 platform. The new pathway relies on a simple operation steps and short detection cycle. From the perspective of the sample amount, BIOCO collector improves the success rate of molecular detection. Our study showed that for about $12 \%$ of cases, it was not possible to obtain enough specimens, thus many patients needed to undergo a second biopsy. The lack of samples may increase the probability of secondary biopsy, the risk of puncture complications, and additional financial burden, as well as reduce the availability of clinical examinations, thus missing the best time for targeted therapy ${ }^{8}$. DNA contamination between specimens is also one of the key factors affecting the accuracy of detection ${ }^{9}$. According to the requirements of LASCA guidelines, the slicing knife should be replaced when slicing the specimen and the knife holder and utensils should be wiped with alcohol to prevent cross-contamination. This further increases the burden of experimenters in practical work. The design of BIOCO solves the problem of sample collection and preservation during the trimming of paraffin blocks, simplifies the follow-up workflow of molecular pathology detection, and reduces cross-contamination.

Compared to the ROUCO collection method, our new BIOCO approach showed similar DNA extraction efficiency. The qualitative results of 39 cases found that one high-abundance patient got a medium-abundance result, and one medium-abundance patient got a low-abundance result. which may be due to the differences in the total amount of DNA collected or the heterogeneity of the tumor. Overall, about $95 \%(37 / 39)$ of the samples obtained consistent abundance, and 100\% (39/39) of the samples obtained consistent mutation detection results; which suggested that the results obtained by BIOCO collected samples were reliable.

However, this study has a few limitations. In order to shorten the period of molecular pathology detection, molecular pathology $\mathrm{H} \& \mathrm{E}$ staining was simultaneously performed with the overnight digestion of the sample. If H\&E staining showed no tumor cells or the content of tumor cells was less than $10 \%$, it did not meet the criteria of pathological quality control, and the samples, reagents, and labor went to waste. However, in practical applications, this occurs very rarely.

\section{Conclusions}

The new diagnosis and treatment pathway accelerates the reporting period of molecular pathology detection, shortens the TAT to 4-6 days, and provides the opportunity for rapid and precise treatment of patients, which has far-reaching implications for each patient and his family. At the same time, the rapid and accurate molecular pathology detection report accelerated the turnover of the hospital beds, improved the efficiency of the use of hospital beds, and reduced the medical expenses. DA8600 platform and BIOCO collector showed to be effective tools for clinical-pathological diagnosis, which can be used by pathology and related departments.

\section{Disclosure}

None declared for all authors. 


\section{Acknowledgment}

This study was supported by the Construction of big data platform for precision medical treatment of common tumors such as lung cancer in Henan Province, Henan Province Major Science and Technology Project (161100311500). We also thank MedSci (https://www.mjeditor.com/) for proofreading the manuscript.

\section{REFERENCES}

1. Cao L, Long L, Li M, et al. The utilization of next-generation sequencing to detect somatic mutations and predict clinical prognosis of Chinese non-small cell lung cancer patients[J]. Onco Targets Ther , 2018; 11:2637-2646.

2. Lung Cancer Group, Respiratory Society, Chinese Medical Association. Expert consensus on molecular targeted therapy for advanced NSCLC [J]. Chinese journal of tuberculosis and respiration, 2014; 37(3): $177-82$.

3. Qingjie H. Analysis of gene mutation phenotype and clinicopathological characteristics of non-small cell lung cancer based on second-generation sequencing [D]. 2019.

4. Lindeman, Neal I, Cagle, et al. Molecular Testing Guideline for Selection of Lung Cancer Patients for EGFR and ALK Tyrosine Kinase Inhibitors: Guideline from the College of American Pathologists, International Association for the Study of Lung Cancer, and Association for Molecular Pathol [J]. Journal of Thoracic Oncology Official Publication of the International Association for the Study of Lung Cancer , 2013; 137(6):828-860.

5. Lindeman N I, Cagle P T, Aisner D L, et al. Updated Molecular Testing Guideline for the Selection of Lung Cancer Patients for Treatment With Targeted Tyrosine Kinase Inhibitors [J]. J Thorac Oncol , 2018; $13(3)$.

6. DiStasio, Marcello, Chen, et al. Molecular Testing Turnaround Time for Non-Small Cell Lung Cancer in Routine Clinical Practice Confirms Feasibility of CAP/IASLC/AMP Guideline Recommendations: A Single-center Analysis [J]. Clin Lung Cancer , 2017; S1525730417300785.

7. Jiang T, Ren S, Li X, et al. The changing diagnostic pathway for lung cancer patients in Shanghai, China [J]. Eur J Cancer, 2017; 84:168-172.

8. Atanesyan L, Steenkamer M J , Horstman A, et al. Optimal Fixation Conditions and DNA Extraction Methods for MLPA Analysis on FFPE Tissue-Derived DNA [J]. AM J Clin Pathol , 2017; 147(1):60-8.

9. Lisong SH, Guohua X. Molecular diagnosis in China: present status and challenges ahead [J]. Chinese Journal of Laboratory Medicine, 2016, 39(7):473-476.

\section{Hosted file}

Fig.1.docx available at https://authorea.com/users/342121/articles/469000-new-protocol-forpathological-diagnosis-and-treatment-pathway-based-on-the-bioco-collector-tool-in-henancancer-hospital

\section{Hosted file}

Fig.2.docx available at https://authorea.com/users/342121/articles/469000-new-protocol-forpathological-diagnosis-and-treatment-pathway-based-on-the-bioco-collector-tool-in-henancancer-hospital

\section{Hosted file}

Fig.3.docx available at https://authorea.com/users/342121/articles/469000-new-protocol-forpathological-diagnosis-and-treatment-pathway-based-on-the-bioco-collector-tool-in-henancancer-hospital 


\section{Hosted file}

FIG.4.docx available at https://authorea.com/users/342121/articles/469000-new-protocol-forpathological-diagnosis-and-treatment-pathway-based-on-the-bioco-collector-tool-in-henancancer-hospital

\section{Hosted file}

TABLE 1.docx available at https://authorea.com/users/342121/articles/469000-new-protocolfor-pathological-diagnosis-and-treatment-pathway-based-on-the-bioco-collector-tool-inhenan-cancer-hospital

\section{Hosted file}

TABLE 2.docx available at https://authorea.com/users/342121/articles/469000-new-protocolfor-pathological-diagnosis-and-treatment-pathway-based-on-the-bioco-collector-tool-inhenan-cancer-hospital

\section{Hosted file}

TABLE 3.docx available at https://authorea.com/users/342121/articles/469000-new-protocolfor-pathological-diagnosis-and-treatment-pathway-based-on-the-bioco-collector-tool-inhenan-cancer-hospital 